\title{
Cooperativas e \\ internacionalización: un análisis de las 300 mayores cooperativas del mundo
}

\author{
Ignacio Bretos \\ Millán Díaz-Foncea \\ Carmen Marcuello
}

RESUMEN: Este artículo aborda un debate clave en el ámbito de la economía social donde la investigación previa es extremadamente limitada: la internacionalización de las cooperativas. Específicamente, presentamos un análisis exploratorio sobre la internacionalización de las 300 mayores cooperativas del mundo entre los años 2010 y 2013, su tamaño y evolución en términos de facturación y empleo, las regiones geográficas donde están localizadas, y los sectores donde desarrollan su actividad. Los datos se han obtenido principalmente del World Cooperative Monitor de la Alianza Cooperativa Internacional y de los informes anuales publicados por las propias cooperativas. La teoría existente sugiere que las cooperativas deberían ser incapaces de expandirse internacionalmente debido a diversas limitaciones e ineficiencias económicas y organizacionales. Sin embargo, nuestros resultados muestran que la internacionalización dista mucho de ser un fenómeno marginal en estas organizaciones. Además, los resultados evidencian que estas cooperativas han aumentado sus niveles de facturación y empleo en el periodo 2010-2013, caracterizado por una severa crisis económica global. En conclusión, este artículo plantea un claro desafío a la teoría actual sobre las cooperativas y advierte la necesidad de revisar nuestra comprensión sobre el rol e impacto de estas organizaciones en el contexto de la globalización.

PALABRAS CLAVE: Cooperativas, crisis económica, Economía Social, empleo, empresa multinacional, globalización, internacionalización, modo de entrada en el mercado extranjero.

CLAVES ECONLIT: F23, F44, E32, J21, P13, J54.

Cómo citar este artículo / How to cite this article: BRETOS, I., DÍAZ-FONCEA, M. \& MARCUELLO, C. (2018):

"Cooperativas e internacionalización: un análisis de las 300 mayores cooperativas del mundo", CIRIEC-España,

Revista de Economía Pública, Social y Cooperativa, 92, 5-37.

Correspondencia: Ignacio Bretos, Millán Díaz-Foncea y Carmen Marcuello, Facultad de Economía y Empresa,

Universidad de Zaragoza. Gran Vía 2, 50005 Zaragoza. E-mail de contacto: ibretos@ unizar.es 


\section{EXPANDED ABSTRACT}

\section{Cooperatives and internationalization: An analysis of the $\mathbf{3 0 0}$ largest cooperatives in the world}

\section{Objectives}

Cooperatives are usually portrayed as small-sized enterprises that operate exclusively at the domestic level. What is more, the literature highlights several inefficiencies of organizational and financial nature that suggest that these organizations should be unable to expand internationally. Among other aspects, it is pointed out that cooperatives are marginal organizations in most countries and industries (Podivinsky and Stewart, 2007), and tend to stay in a small size due to their democratic nature, community orientation, and social purposes, which may become more complex to promote as these organizations grow and scale up their operations (Rothschild-Whitt, 1986). It is also suggested that cooperatives suffer from various disadvantages, including the lack of funding and investment capacity in these organizations (Van der Krogt et al., 2007), the difficulties in establishing efficient decisionmaking mechanisms (Österberg and Nilsson 2009), and the limitations to attract and retain competent and valuable managers (Morales 2004).

In view of this, the article aims to test if there are internationalized cooperatives in the current globalized world, as well as to analyse their degree of internationalization (based on the foreign market entry strategy), their size in terms of turnover and employment, their home countries, and the sectors in which they operate.

\section{Methodology}

To address our research questions, we provide an exploratory analysis about the internationalization of the 300 largest cooperatives in the world between 2010 and 2013. The study draws on various sources. The classification of the 300 largest cooperatives in the world has been obtained from the "World Cooperative Monitor" reports published by the International Cooperative Alliance and Euricse (https://monitor.coop/). These reports also provide information about the country of origin of the cooperatives, the main sector of activity in which they operate, and their turnover figures (expressed in US dollars). The following sectors are differentiated: Agriculture and food; Industry and utilities; Wholesale and retail trade; Insurance; Banking and financial services; Health, education and social care; and Other services. Meanwhile, the data on employment and internationalization have been chiefly obtained from the information disclosed by each of the cooperatives (annual reports, sustainability reports, and financial reports). 
Following the literature on foreign market entry mode (e.g., Brouthers and Hennart, 2007), we analyse the degree of internationalization of the cooperatives by identifying the strategy used to access foreign markets. Four main modes are distinguished, on a scale from lower to higher degree of internationalization in terms of control, return, risk, and resources (Johanson and Vahlne, 2009):

- Domestic: the cooperative operates exclusively at the domestic level, that is to say, it does not carry out any type of transaction in a foreign market.

- Export entry mode: the cooperative only carries out exports to foreign countries (minimum degree of internationalization).

- Contractual agreement entry modes: the cooperative operates in foreign markets through licenses or franchises (medium degree of internationalization).

- Equity entry modes: the cooperative operates at a global scale through joint ventures or wholly owned subsidiaries (maximum degree of internationalization).

\section{Results/Limitations/Implications}

Our extensive review of the literature about business internationalization and the cooperative's economic and organizational characteristics identified five key aspects that should constrain the ability of these organizations to expand across national borders: (1) the limited creation of cooperatives and the marginal dimension of the cooperative sector in most countries and industries; (2) the inability and reluctance of cooperatives to grow; (3) the difficulties of cooperatives in attracting and retaining trained and experienced managers; (4) the inefficient decision-making mechanisms of cooperatives; and (5) the limited financial and investment capacity of cooperatives.

However, our empirical findings challenge these theoretical predictions drawn from the previous literature, suggesting that the international expansion of cooperatives is a far from marginal phenomenon. Roughly $85 \%$ of the 300 largest cooperatives in the world operate across borders. Moreover, the high degree of internationalization of these cooperatives is evident. About $55 \%$ of them are present in foreign markets through equity agreements. $20 \%$ of the cooperatives operate globally through contractual agreements, whereas $10 \%$ only carries out exports.

In addition, our findings illustrate the positive behaviour of cooperatives in situations of recession (Bretos and Morandeira 2016). The 300 largest cooperatives in the world have increased their turnover and employment levels between 2010 and 2013; a period characterized by a severe global economic crisis. It is also observed that the internationalized cooperatives perform better in terms of employment and turnover during this period than the domestic cooperatives. In particular, the internationalized cooperatives as a whole reached in 2010 a turnover close to USD 1,500 million and employed more than 2 million workers. In 2013, these figures increased to USD 1,900 million and 2.3 million jobs. These results appear to be consistent with those of previous studies demonstrating that global companies have better capacities and greater flexibility to withstand situations of economic crisis (Varum and Rocha, 2011). 
The exploratory nature of this study involves some limitations while, at the same time, opens new avenues for further research. Firstly, the small size of the sample prevents us from providing a more precise and detailed image about the scale of the phenomenon of internationalization in cooperatives. In addition, the fact that the database is made up of the largest cooperatives in the world surely implies an oversizing of the proportion of internationalized cooperatives. Secondly, our study demonstrates that there are internationalized cooperatives, but there is still a long way to advance our knowledge about how cooperatives internationalize. For example, how internationalized cooperatives faced their limitations to attract and retain valuable managers or to have investment capacity? Future studies could test the five hypotheses proposed in our theoretical framework in order to better understand the internationalization of cooperatives. Another key issue is to refine our knowledge about the internationalization strategies deployed by cooperatives. The way we have had to collect the data has not allowed us to distinguish between greenfield investment and joint venture, although there are important differences between these strategies in terms of control, return, risk, and resources commitment. Therefore, moving forward in this direction may enrich our understanding about the degree of internationalization of cooperatives.

\section{Conclusions/originality}

This article addresses a key debate in the Social Economy field about which previous research is extremely limited: the internationalization of cooperatives. Our findings question several assumptions of the extant theory on cooperatives, which suggest that these organizations are inefficient in organizational and economic terms and unable to survive and grow in a global context. The evidence gathered from the analysis demonstrates that there are large cooperatives operating beyond their national boundaries, and that they compete effectively in the main worldwide economies and industries. Accordingly, in line with some previous studies (Bretos and Marcuello, 2017; Pérotin, 2015), we argue for revising and reconsidering our view about the role and impact of cooperatives in the context of globalization.

KEYWORDS: Cooperatives, economic crisis, Social economy, employment, multinational company, globalization, internationalization, foreign market entry mode. 


\section{Introducción 1}

Tradicionalmente, las cooperativas han sido representadas en la literatura como organizaciones pequeñas que operan exclusivamente a nivel doméstico, debido a sus características organizacionales y a su enraizamiento social en los territorios y comunidades locales (Pérotin 2015). No obstante, una dinámica clave que ha definido la intensificación del proceso de globalización en las tres últimas décadas ha sido la creciente expansión internacional no sólo de grandes corporaciones, sino también de muchas pequeñas y medianas empresas (Acs et al. 2001). En tanto que las cooperativas no han sido ajenas a las presiones y tendencias de la globalización, parece lógico argumentar que muchas de estas organizaciones han podido decidir o verse obligadas a crecer más allá de sus fronteras nacionales. Sin embargo, es evidente que nuestro conocimiento sobre el fenómeno de la internacionalización empresarial en el caso de las cooperativas es extremadamente limitado, tal y como corroboran algunas revisiones recientes de la literatura (Bretos y Marcuello 2017).

Hasta ahora, sólo un número muy reducido de estudios han mostrado que existen cooperativas operando más allá de sus fronteras nacionales, tales como la cooperativa alimentaria neozelandesa Fonterra, la cooperativa bancaria holandesa Rabobank, la cooperativa distribuidora escandinava Coop Nord y, particularmente, las cooperativas industriales del grupo Mondragón (Birchall 2014). En este caso, un amplio número de estudios han analizado cuestiones muy diversas, tales como las contradicciones generadas por la expansión internacional en Mondragón (Bretos y Errasti 2018; Errasti et al. 2016), la cooperativización de algunas de sus filiales capitalistas en el ámbito doméstico (Bretos y Errasti 2016, 2017), las políticas y prácticas de gestión internacional de recursos humanos (Bretos et al. 2018a, 2018b), o la caída de Fagor Electrodomésticos, el buque insignia del grupo, con cerca de 11.000 trabajadores en sus plantas en España, Francia, China, Polonia, Marruecos e Italia (Errasti et al. 2017).

De hecho, en términos generales, la literatura destaca diversas ineficiencias de carácter económico y organizativo en las cooperativas que sugieren que estas organizaciones no son capaces de crecer internacionalmente y que, por tanto, no deberían existir cooperativas transnacionales. Entre otros aspectos, se señala que las cooperativas son marginales en la mayoría de países y sectores de actividad (Podivinsky y Stewart, 2007) y tienden a permanecer en una reducida dimensión (Dow 2003). Asimismo, se sugiere que las cooperativas adolecen de diversas desventajas como, por ejemplo, las dificultades para establecer mecanismos eficientes en la toma de decisiones (Österberg y Nilsson 2009), las limitaciones para atraer y retener directivos competentes y valiosos (Morales 2004) o la problemática derivada de la falta de financiación e inversión en estas organizaciones (Van der Krogt et al., 2007).

1.- Los autores agradecen el apoyo recibido desde el Instituto Euricse (European Research Institute on Cooperative and Social Enterprises) en la recolección de los datos, así como los comentarios y sugerencias de Virginie Pérotin, Juan Francisco Juliá, Saioa Arando y Gabriel Burdín. Cualquier error u omisión que pudiese contener el artículo es responsabilidad exclusiva de los autores. 
Por tanto, el objetivo de este estudio es contrastar si existen cooperativas internacionalizadas, analizar su grado de internacionalización (atendiendo a la modalidad de presencia en los mercados extranjeros), su tamaño en términos de facturación y empleo, las regiones geográficas de donde provienen y los sectores de actividad donde están presentes. Para abordar estas cuestiones, presentamos un análisis exploratorio sobre las 300 mayores cooperativas del mundo entre los años 2010 y 2013, en base a los informes "World Cooperative Monitor" elaborados por la Alianza Cooperativa Internacional y el Instituto Euricse y a los datos recogidos en los informes anuales y otros documentos publicados por las propias cooperativas.

Tras esta introducción, el siguiente aprtado contextualiza brevemente el proceso de internacionalización empresarial y los principales modos de entrada en los mercados extranjeros. El tercer apartado conecta la literatura sobre la internacionalización con las características económicas y organizacionales de las cooperativas para establecer una serie de hipótesis. El cuarto apartado detalla la metodología y datos empleados. El quinto apartado presenta los resultados obtenidos en el análisis. Finalmente, se extraen las conclusiones del artículo, discutiendo también sus limitaciones y proponiendo algunas posibles líneas para la investigacion futura.

\section{La internacionalización empresarial: Una breve contextualización}

Un factor fundamental que explica la internacionalización de las empresas es la creciente intensificación del proceso de globalización. En el ámbito económico-productivo, este fenómeno implica un proceso de apertura y movilidad del capital asociado a múltiples procesos de integración económica regional que expone los tejidos productivos nacionales a una competencia exterior creciente (Bretos y Marcuello 2017). Ello se traduce en una conectividad internacional e interdependencia de las economías nacionales, lo que supone que los competidores, proveedores y clientes de una empresa pueden encontrarse en cualquier lugar del mundo.

De manera más específica, la globalización contiene tres fuerzas que promueven la internacionalización de las empresas (Acs et al. 2001): (1) el crecimiento extraordinario de las tecnologías de bajo coste que conectan personas y lugares; que favorece la división de los procesos productivos de las empresas; (2) la reducción constante de las barreras comerciales, que han generado un ámbito de actuación cada vez más amplio para las empresas; y (3) la reestructuración económica y liberalización que se ha producido, creando así nuevas oportunidades para la inversión y el crecimiento. De acuerdo con la literatura, la internacionalización puede proveer de importantes ventajas a las empresas. Entre ellas destacan la mayor dimensión de la empresa y su mayor poder de mercado sobre distribuidores, proveedores y clientes, la obtención de economías de escala y alcance, la reducción del 
riesgo a través de la diversificación internacional o la posibilidad de desplazar la producción ante cambios en las condiciones de los mercados, la accesibilidad a diferentes conocimientos, aprendizajes y capacidades en países extranjeros, las ventajas por localizarse en regiones con menores costes y otras ventajas específicas de un país extranjero que pueden hacer que una empresa establezca allí una filial para explorar esas ventajas y mejorar su competitividad tanto en dicho país como en el suyo de origen (véase Sapienza et al, 2006 para una revisión).

La internacionalización empresarial puede ser definida como un proceso por el que una empresa aumenta gradualmente su implicación en operaciones internacionales. Este proceso evoluciona en una interacción entre, por un lado, el desarrollo del conocimiento sobre los mercados extranjeros y las operaciones y, por otro lado, el compromiso creciente de recursos en los mercados extranjeros. Así, se argumenta que la mayoría de empresas tienden a expandirse de manera secuencial en una serie de etapas que conllevan un mayor grado de implicación en sus operaciones internacionales (Johanson y Valhne, 1977, 2009). Asimismo, una amplia línea de investigación se ha centrado en estudiar los diferentes modos de entrada de las empresas en los mercados extranjeros. Sharma y Erramilli (2004: 2) definen el modo de entrada como 'un acuerdo estructural que permite a una empresa implementar su estrategia de mercado del producto en un país extranjero, ya sea mediante la realización exclusiva de operaciones de comercialización (es decir, a través de la exportación), o mediante la realización de operaciones de producción y comercialización, bien por sí misma o en colaboración con otros (jointventures, filiales propias...).' Si bien algunos trabajos han identificado multitud de variantes en el modo de entrada al extranjero (para una detallada revisión, véase Brouthers y Hennart 2007), en términos generales, pueden distinguirse en 3 grandes modalidades: exportaciones; acuerdos contractuales; $y$ acuerdos accionariales. Estas estrategias difieren en torno a cuatro factores esenciales: riesgo, rendimiento, recursos y control (Agarwal y Ramaswami 1992).

Cada uno de los modos de entrada se relacionan distintamente con estos cuatro factores (véase el Cuadro 1 para un resumen). El modo de entrada mediante exportaciones es una alternativa que supone un bajo compromiso de recursos (inversión) y, por tanto, un bajo riesgo y rendimiento, que otorga a la empresa un control operacional pero no proporciona un control del mercado. Los modos de entrada mediante acuerdos accionariales (joint venture y filial propia) se sitúan en el extremo opuesto. Por un lado, la entrada a un mercado extranjero con una filial propia es una alternativa de inversión elevada y, consecuentemente, de elevado riesgo y rendimientos, que además proporciona a la empresa inversora un elevado grado de control. Por otro lado, la joint venture es una alternativa que supone relativamente un menor compromiso de recursos y, por tanto, proporciona un riesgo, rendimiento y control acorde al grado de participación en el capital de la empresa inversora. Finalmente, los modos de entrada mediante acuerdos contractuales (principalmente licencias, concesiones y franquicias) se sitúan en una posición intermedia, conllevando una menor inversión, riesgo, rendimiento y control que los acuerdos accionariales, pero mayor que en el caso de las exportaciones (Agarwal y Ramaswami 1992; Brouthers y Hennart 2007). 


\section{Cuadro 1. Principales modos de entrada en los mercados extranjeros}

\begin{tabular}{|l|l|l|}
\hline Modo de entrada & Tipologías & Implicaciones \\
\hline Exportación & Directas e Indirectas & Menor inversión, riesgo, rendimiento y control \\
\hline Acuerdos contractuales & Licencias, concesiones y franquicias & $\begin{array}{l}\text { Nivel intermedio de inversión, riesgo, rendimiento } \\
\text { y control }\end{array}$ \\
\hline Acuerdos accionariales & Joint-venture & $\begin{array}{l}\text { Nivel de inversión, riesgo, rendimiento y control altos, } \\
\text { acorde al grado de participación en el capital }\end{array}$ \\
\cline { 2 - 3 } & $\begin{array}{l}\text { Filial propia (mediante greenfield } \\
\text { investment o adquisición/absorción) }\end{array}$ & Máxima inversión, riesgo, rendimiento y control \\
\hline
\end{tabular}

FUENTE: adaptado de Brouthers y Hennart (2007)

\section{Las cooperativas y la expansión internacional: Predicciones de la literatura}

En este apartado se conecta la literatura sobre la internacionalización empresarial con la teoría existente acerca de las características organizacionales y económicas de las cooperativas (véase un resumen en el Cuadro 2). A partir de esta extensa revisión de la literatura, se han identificado 5 factores clave que permiten sugerir que las cooperativas no deberían ser capaces de expandirse internacionalmente: (1) la creación limitada de cooperativas y la dimensión marginal del sector cooperativo en la mayoría de países y sectores de actividad; (2) las desventajas de las cooperativas derivadas de su reducido tamaño; (3) las dificultades de las cooperativas para contar con una dirección empresarial capacitada; (4) los mecanismos ineficientes de las cooperativas para la toma de decisiones; y (5) las limitaciones financieras de las cooperativas para llevar a cabo las elevadas inversiones. 


\section{Cuadro 2. Limitaciones de las cooperativas para expandirse internacionalmente}

\begin{tabular}{|l|l|l|}
\hline $\begin{array}{l}\text { Características de las } \\
\text { cooperativas }\end{array}$ & Dificultades para la internacionalización & $\begin{array}{l}\text { Citas bibliográficas } \\
\text { representativas }\end{array}$ \\
\hline $\begin{array}{l}\text { Creación limitada de } \\
\text { cooperativas y dimensión } \\
\text { marginal del sector coop. }\end{array}$ & $\begin{array}{l}\text { Carencia de las instituciones formales e informales adecuadas } \\
\text { para establecer cooperativas en muchos países extranjeros. }\end{array}$ & $\begin{array}{l}\text { Pérotin 2006; Podivinsky y Stewart 2007; } \\
\text { Arando et al. 2009; Díaz-Foncea y } \\
\text { Marcuello 2015 }\end{array}$ \\
\hline $\begin{array}{l}\text { Tamaño reducido de las } \\
\text { cooperativas }\end{array}$ & $\begin{array}{l}\text { Desventajas derivadas del tamaño reducido para crecer (caren- } \\
\text { cias der recursos gerenciales, limitaciones financieras, dificul- } \\
\text { tades para alcanzar economías de escala, etc.) }\end{array}$ & $\begin{array}{l}\text { Vanek 1977; Ben-ner 1988; } \\
\text { Rothschild-Whitt 1986; } \\
\text { Cornforth y Thomas 1990; Dow 2003 }\end{array}$ \\
\hline $\begin{array}{l}\text { Dificultades para atraer y y } \\
\text { retener directivos valiosos }\end{array}$ & $\begin{array}{l}\text { Carencia de una dirección capacitada para desenvolverse en } \\
\text { un contexto de competencia internacional (falta de experiencia } \\
\text { internacional, asignación ineficiente de recursos, etc.) }\end{array}$ & $\begin{array}{l}\text { Cornforth y Thomas 1990; Morales 2004; } \\
\text { Spear 2004; Chaves y Sajardo 2004 }\end{array}$ \\
\hline $\begin{array}{l}\text { Procesos ineficientes de } \\
\text { toma de decisiones }\end{array}$ & $\begin{array}{l}\text { Ineficiencias en la toma de decisiones en un entorno interna- } \\
\text { cional complejo y cambiante (falta de liderazgo, toma lenta de } \\
\text { decisiones clave, etc.) }\end{array}$ & $\begin{array}{l}\text { Rothschild-Whitt 1986; Hansmann 1996; } \\
\text { Chaddad y Cook 2004; Österberg y } \\
\text { Nilsson 2009 }\end{array}$ \\
\hline $\begin{array}{l}\text { Estructura financiera débil } \\
\text { e infra-inversión }\end{array}$ & $\begin{array}{l}\text { Incapacidad para abordar la elevada inversión y riesgo que } \\
\text { supone desarrollar estrategias de internacionalización. }\end{array}$ & $\begin{array}{l}\text { Jensen y Meckling 1979; Dow 2003; Van } \\
\text { der Krogt et al. 2007; } \\
\text { Österberg y Nilsson 2009 }\end{array}$ \\
\hline
\end{tabular}

FUENTE: Elaboración propia.

\subsection{Creación de cooperativas y dimensión del sector cooperativo}

La literatura sostiene que la presencia y creación de cooperativas es marginal en relación con la de empresas capitalistas en la mayoría de sectores de actividad y países (Ben-ner, 1988) debido a diversos factores. En primer lugar, se señala que las cooperativas se crean fundamentalmente en etapas cortas caracterizadas por la recesión económica y el desempleo debido a su carácter anticíclico, desapareciendo en etapas de recuperación o crecimiento económico (Pérotin, 2006; Arando et al., 2009). Asimismo, se sugiere que la creación de cooperativas depende de la existencia de instituciones formales e informales en mayor medida que las empresas capitalistas. Por ejemplo, algunos trabajos (Ben-ner, 1988; Arando et al., 2012; entre otros) destacan que las cooperativas requieren de la existencia de instituciones formales adecuadas que proporcionen servicios específicos como asesoramiento y experiencia legal o financiación, debido a la escasez de información sobre los aspectos legales, organizacionales y financieros específicos de estas organizaciones. Asimismo, se asume que la creación de cooperativas se producirá en una región o sector donde exista previamente una elevada densidad cooperativa, debido a la menor legitimidad institucional de la fórmula cooperativa y las barreras de entrada generadas por la escasez de información acerca de estas organizaciones (Arando et al., 2012; Díaz-Foncea y Marcuello, 2015). 
Del mismo modo, la literatura sostiene que las cooperativas tienden a estar presentes en sectores intensivos en manos de obra, con procesos productivos y tecnologías simples, y caracterizados por la presencia mayoritaria de empresas de tamaño reducido (Dow, 2003). Podivinsky y Stewart (2007) señalan que el tamaño del sector cooperativo y la entrada de nuevas cooperativas en las economías de mercado es marginal debido a que las cooperativas enfrentan problemas particulares en la financiación de inversión y en la distribución del riesgo. De esta manera, concluyen que la entrada de cooperativas es residual en sectores altamente intensivos en capital y caracterizados por la presencia de un elevado riesgo. Asumiendo que nos encontramos en un entorno empresarial cada vez más global, desregulado, competitivo y capital-intensivo, la presencia de cooperativas debería ser extremadamente limitada (Chaddad y Cook, 2004).

Todos estos factores se traducen en que las cooperativas deberían ser prácticamente inexistentes en la mayoría de países y sectores de actividad, dificultando así su expansión internacional por diversos motivos. Por un lado, las cooperativas pueden tener dificultades para acceder a mercados extranjeros, debido a que en muchos países no existen instituciones formales adecuadas para la constitución de cooperativas (por ejemplo, por la carencia de legislación cooperativa) ni instituciones informales (por ejemplo, por la falta de cultura y tradición cooperativista) (Bretos et al. 2018a, 2018b). De esta forma, la expansión internacional de una cooperativa, por ejemplo, mediante la implantación de una nueva filial (greenfield investment) se vería limitada por la complejidad a la hora de implantar el modelo cooperativo en un país extranjero. Por otro lado, debido a la escasa presencia de cooperativas en la mayoría de sectores y países, se presume que las cooperativas tendrán dificultades para encontrar otras cooperativas socias con las que establecer alianzas (joint ventures) o realizar fusiones (Flecha y Ngai 2014). En muchas ocasiones, dichas estrategias son esenciales para acceder a ciertos mercados extranjeros debido a las diferencias culturales, distancia institucional o necesidad de compartir riesgos y costes (Barkema y Vermeulen, 1997).

\subsection{Tamaño de las cooperativas}

La literatura sugiere que las cooperativas suelen crearse con un menor tamaño que las empresas capitalistas y tienen un patrón de crecimiento mucho más lento (Ben-ner 1988). Uno de los principales factores señalados es que las cooperativas tienden a permanecer en una reducida dimensión debido a razones sociales como el mantenimiento del nivel de participación democrática en la organización y de su orientación comunitaria, en tanto que el mayor tamaño de la cooperativa puede menoscabar la involucración colectiva en la toma de decisiones (Rothschild-Whitt, 1986; Dow 2003). Otros trabajos sugieren que las cooperativas no tienen los mismos incentivos para crecer indefinidamente que las empresas capitalistas, ya que la maximización del beneficio por cada miembro puede ser independiente de la escala (Vanek, 1977). Igualmente, el reducido tamaño de las cooperativas se ha atribuido a sus diferentes desventajas y limitaciones financieras. Algunos trabajos también señalan que las cooperativas tienen limitaciones para crecer a través de adquisiciones y absorciones con otras empresas ya que no pueden obtener capital mediante la emisión de acciones u ofrecer un intercam- 
bio de acciones (Cornforth y Thomas, 1990). Finalmente, se destaca que, debido a su escasa presencia en la mayoría de países y sectores, las cooperativas tienen grandes dificultades para encontrar otras cooperativas socias con las que establecer alianzas y adquirir un mayor tamaño (Flecha y Ngai, 2014).

La reducida dimensión de las cooperativas plantea problemas para su expansión internacional. Como señalan diversos trabajos (Yip et al. 2000; Lu y Beamish 2001; Qian, 2002), las empresas pequeñas sufren diversas limitaciones para expandirse internacionalmente. Por un lado, es habitual que estas empresas no cuenten con directivos especializados en la gestión internacional o con un mando jerárquico a través del que tomar las decisiones adecuadas en el ámbito internacional, lo que puede llevar a que estas empresas no dispongan de la información necesaria para aprovechar oportunidades en los mercados internacionales (Qian, 2002). Asimismo, la literatura ha destacado que, debido a sus limitaciones para obtener recursos, las empresas pequeñas asumen unos niveles de riesgo más altos que otras empresas de mayor tamaño ya que deberán destinar una proporción de recursos mucho mayor (Lu y Beamish, 2001). Igualmente, estas empresas sufren desventajas respecto a sus competidores a la hora de obtener economías de escala, lo que afecta negativamente a la probabilidad de éxito de sus iniciativas de internacionalización (Yip et al., 2000).

\subsection{Gestión y dirección en las cooperativas}

La literatura destaca que las cooperativas enfrentan diversas dificultades a la hora de atraer y retener directivos valiosos (Morales 2004). Entre otras cuestiones, ello se ha atribuido a las limitaciones salariales existentes en las cooperativas, ya que suelen pagar salarios más bajos a sus directivos en comparación con las empresas capitalistas y, además, las diferencias salariales entre directivos y otros miembros con menor grado de responsabilidad son mucho más reducidas (Spear, 2004). Igualmente, se señala que, debido al reducido tamaño de las cooperativas, éstas no pueden habitualmente ofrecer los mismos incentivos materiales ni carreras profesiones tan atractivas a los directivos que las empresas capitalistas (Cornforth y Thomas, 1990). En una línea similar, algunos trabajos sugieren que el continuo escrutinio y control sobre los directivos en las cooperativas también influye a la hora de atraer y retener directivos valiosos en estas organizaciones (Morales, 2004). Cornforth y Thomas (1990) también destacan que, dado que el sector cooperativo es muy reducido en la mayoría de países, no existe un mercado laboral de directivos cooperativistas. Ello se traduce en que, habitualmente, los directivos que entran en las cooperativas provengan de empresas capitalistas, existiendo una problemática derivada de la falta de especialización de los directivos en la cultura y valores cooperativistas (Spear, 2004; Chaves y Sajardo, 2004).

Las dificultades para atraer y retener directivos valiosos también tienen implicaciones para la capacidad de las cooperativas de expandirse internacionalmente. La figura del directivo ha sido considerada como crucial en las empresas para crear valor y generar ventajas competitivas sostenibles en el contexto competitivo actual, ya que juegan un papel fundamental en el análisis del entorno, en la 
definición de la estrategia de la empresa, en la combinación de recursos que se utilizarán en cada etapa, y en la elección de los mercados donde la empresa competirá (Acquaah, 2003). Estos aspectos son todavía más críticos en el caso de una empresa internacionalizada, ya que debe desenvolverse en un contexto económico y empresarial más complejo, cambiante y dinámico (Hitt et al., 2006). Por ejemplo, Sapienza et al. (2006) han destacado el papel fundamental de los directivos con experiencia internacional y altas capacidades en el rendimiento de las empresas internacionalizadas, siendo especialmente crítico en las fases tempranas de la internacionalización.

\subsection{Toma de decisiones en las cooperativas}

La literatura también subraya diversas ineficiencias relacionadas con la toma de decisiones colectiva y democrática en las cooperativas, al suponer unos costes superiores que en las empresas capitalistas (Rothschild-Whitt, 1986). Los costes de utilizar mecanismos de decisión colectiva para poner en común las preferencias de los miembros de una cooperativa y transformarlas en acciones son notablemente superiores que en el caso de una empresa capitalista (Österberg y Nilsson 2009). Ello se basa en que la disparidad y heterogeneidad de los miembros de una cooperativa elevan el coste de la toma de decisiones colectiva. Como señala Hansmann (1996), para llevar a cabo una toma de decisiones colectiva de manera apropiada e implantar dichas decisiones de forma efectiva, los miembros de la cooperativa deben invertir una cantidad significativa de tiempo y esfuerzo en conocer la empresa y las preferencias de los otros miembros, por ejemplo, a través de la comunicación o de la asistencia a reuniones y asambleas. Asimismo, diversos trabajos han asociado la democracia organizacional e igualitarismo con la lentitud en la toma de decisiones y con la anulación de los roles de liderazgo en las personas de la organización; aspectos que pueden ser determinantes para la supervivencia y devenir de la cooperativa (Chaddad y Cook 2004).

Ello sugiere que la toma de decisiones en las cooperativas puede ser ineficiente, lenta y costosa. Estos aspectos son esenciales en una empresa que opera a nivel internacional, ya que la creciente complejidad organizativa y los mayores flujos de información implican un aumento en los costes del proceso de toma de decisiones y, por tanto, requieren mecanismos eficientes para procesar la información y tomar las decisiones adecuadas en base a una relación causa-efecto que maximice el valor de la empresa (Luo, 2005). Esto requiere en muchas ocasiones que la toma de decisiones estratégicas sea llevada a cabo por un grupo reducido de personas (Sanders y Carpenter, 1998). Asimismo, en entornos competitivos dinámicos, la toma de decisiones ágil es un factor clave que determina el éxito de las empresas (Joyce y Woods, 2003). Esto resulta crítico en una empresa que opera a nivel internacional, ya que debe desenvolverse a un entorno cambiante y complejo que requiere agilidad y liderazgo a la hora de tomar de decisiones clave (Kedia et al., 2002). 


\subsection{Estructura de capital e inversión en las cooperativas}

La literatura también se ha centrado en la problemática que plantea la particularidad de los derechos de propiedad en las cooperativas, sosteniendo que deriva en limitaciones para acceder a capital y en una estructura financiera débil (Ben-Ner, 1988; Van der Krogt et al., 2007). Se señala que la estructura de propiedad de las cooperativas da lugar a fallos organizativos y a decisiones ineficientes sobre empleo e inversiones, lo que puede afectar de manera negativa a la supervivencia de las cooperativas en comparación con las empresas de capital (Jensen y Meckling, 1979). En relación a las decisiones de inversión, se señala que las cooperativas sufren el 'problema del horizonte', es decir, que los socios evalúan únicamente proyectos de inversión dentro del horizonte temporal en el que esperan estar empleados (Österberg y Nilsson 2009). Esto se traslada al problema de la 'infra-inversión', el cual supone que los socios preferirán repartir las rentas en el periodo actual en lugar de dedicarlo a financiar inversiones futuras (Vanek, 1977). Asimismo, la literatura ha destacado que la aversión al riesgo es mayor entre los socios de las cooperativas, implicando que éstos sólo realizarán inversiones cortoplacistas con una reducida probabilidad de fracaso (Jensen y Meckling, 1979), lo que lleva a una menor propensión a la inversión e innovación por parte de las cooperativas. Finalmente, otros trabajos señalan que las cooperativas tienen limitaciones para acceder a fuentes de financiación externa debido, por ejemplo, a que los prestamistas suelen percibir un mayor riesgo en las cooperativas 0 a que impondrán condiciones más duras de financiación al no poder tomar parte en el control de la cooperativa (Dow 2003).

Las limitaciones de las cooperativas para acceder al capital y disponer de financiación implican serias desventajas para que estas organizaciones se internacionalicen. En este sentido, como ha demostrado diversa evidencia empírica (Johanson y Valhne, 2009; entre otros), las estrategias de internacionalización de alto compromiso, como el establecimiento de filiales en el extranjero, implican un compromiso elevado de recursos y requieren asumir grandes riesgos, cuestión que puede verse impedida por una estructura de propiedad como la de las cooperativas (George et al. 2005). En esta línea, para crecer internacionalmente es fundamental tener una fuerte capacidad de inversión y poder contar con fuentes de financiación externa (Yip et al. 2000; Lu y Beamish 2001). 


\section{Metodología y datos}

El estudio se basa en diversas fuentes de información. En primer lugar, se han utilizado los informes denominados World Cooperative Monitor que elabora anualmente desde el año 2012 la Alianza Cooperativa Internacional en colaboración con el Instituto Euricse (http://www.euricse.eu/). Se han analizado cuatro informes (ICA, 2012, 2013, 2014, 2015), los cuales proporcionan información con un retardo temporal de dos años, es decir, los datos hacen referencia a los años 2010, 2011, 2012 y 2013. En concreto, estos informes proporcionan una lista con las 300 mayores cooperativas del mundo en cada uno de los años (es decir, 1.200 observaciones durante los cuatro años), así como información relativa a su país de origen, sector principal de actividad, y cifras de facturación. El orden en la lista de las 300 mayores cooperativas del mundo se ofrece en función de su facturación, expresada en dólares americanos. El país de origen de las cooperativas se basa en la clasificación "Standard Country or Area Codes for Statistical Use" elaborada por las Naciones Unidas. En relación al sector de actividad, se diferencian los siguientes:

- Comercio mayorista y minorista: Incluye las cooperativas constituidas para comprar y proveer bienes y servicios en condiciones más competitivas en interés de sus socios. Fundamentalmente, son cooperativas de consumo.

- Agricultura y alimetación: Agrupa a las cooperativas que operan a lo largo de toda la cadena de valor agrícola, desde el cultivo de productos agropecuarios y la ganadería hasta la elaboración industrial de productos agrícolas y animales. Este sector incluye cooperativas de productores agrícolas y consorcios de cooperativas que llevan a cabo la transformación y comercialización de productos agrícolas para sus miembros.

- Industria: Incluye las cooperativas que operan en sectores industriales (a excepción de la industria alimentaria) y cooperativas que gestionan la infraestructura de un servicio público como la electricidad, gas y agua. Este sector también incluye cooperativas de trabajo en el sector manufacturero.

- Servicios bancarios y financieros: Incluye bancos cooperativos y cooperativas de crédito que prestan servicios de intermediación bancaria y financiera, controlados democráticamente por los miembros (prestatarios y depositantes).

- Seguros: Agrupa mutuas y cooperativas que son propiedad de sus clientes asegurados y que están gestionadas democráticamente por ellos. Estas entidades permiten a los miembros obtener pólizas de seguros en condiciones más favorables que las disponibles en el mercado.

- Asistencia sanitaria y social: Incluye cooperativas que gestionan servicios de salud, sociales y educacionales. Ello puede comprender cooperativas de consumidores/usuarios, cooperativas productoras, y cooperativas que buscan proveer servicios sociales y de salud de mayor calidad y/o a un menor coste. 
- Otros servicios: Abarca todas las cooperativas que prestan servicios distintos de los incluidos en la atención sanitaria y social, como los servicios cooperativos de negocios y transporte.

Esta información se ha complementado con los datos de empleo y del grado de internacionalización de las 300 mayores cooperativas del mundo en cada uno de los años comprendidos en el periodo 2010-2013. Los datos de empleo se han obtenido principalmente de la información divulgada de manera pública por cada una de las cooperativas (fundamentalmente se han empleado los informes anuales, memorias de sostenibilidad e informes financieros). Asimismo, también se ha utilizado la base de datos Amadeus para obtener las cifras de empleo de algunas cooperativas europeas (alrededor del 15\% de la muestra de cooperativas europeas). Se han logrado obtener las cifras de empleo para 594 cooperativas sobre el total de la muestra de 1.200 entre 2010 y 2013 , es decir, un 49,5\%. En concreto, se dispone de cifras de empleo para el $49,67 \%$ del total de las 300 mayores cooperativas del mundo en el año 2010; para el 47,67\% en 2011; para el 48,67\% en 2012; y para el 52\% en 2013.

En relación al grado de internacionalización, los datos se han extraído principalmente de los informes anuales y las memorias de sostenibilidad publicadas por cada una de las cooperativas. Nuevamente, no se han podido obtener datos sobre el grado de internacionalización para todas las cooperativas. En 2010 se dispone de datos del 69\% de cooperativas; en 2011 del 72\%; en 2012 también del $72 \%$; y en 2013 del 73\%. Para analizar el grado de internacionalización de las cooperativas, se ha identificado la estrategia empleada por cada una de las cooperativas para estar presentes en los mercados extranjeros. Siguiendo la clasificación de los modos de entrada en los mercados internacionales establecida en el anterior Cuadro 1, la dimensión internacional de las cooperativas se ha diferenciado en 4 modalidades, en una escala de menor a mayor grado de compromiso internacional (Johanson y Vahlne, 1977, 2009):

- No internacionalización: la cooperativa desarrolla su actividad exclusivamente a nivel nacional, es decir, no lleva a cabo ningún tipo de transacción en un mercado extranjero.

- Exportación: la cooperativa lleva a cabo únicamente exportaciones a los países extranjeros. Mínimo grado de internacionalización.

- Acuerdos contractuales: la cooperativa está presente en los mercados extranjeros a través de acuerdos contractuales (licencias, concesiones o franquicias). Grado de internacionalización intermedio.

- Acuerdos accionariales: la cooperativa está presente en los mercados extranjeros a través de acuerdos accionariales (joint-ventures y filiales propias, ya sean comerciales o productivas). Máximo grado de internacionalización.

Cabe señalar que la literatura realiza importantes matizaciones entre las distintas estrategias dentro de cada modalidad. Por ejemplo, la exportación indirecta (a través de intermediarios) implica menores niveles de riesgo, control y compromiso de recursos en comparación con la exportación directa a los clientes en el extranjero (Fernández-Olmos y Díez-Vial, 2014). Asimismo, en el ámbito de los acuerdos accionariales, la filial propia es el modelo que implica un mayor grado de internacionalización, 
mientras que las alianzas suponen un grado de control, inversión, riesgo y rendimiento comparativamente menor; acorde al grado de participación en el capital de la empresa inversora (Brouthers y Hennart, 2007). No obstante, estas diferenciaciones quedan fuera del alcance de este estudio. En muchas ocasiones, las memorias no recogían la distinción entre exportación directa o indirecta. Además, muchas cooperativas no especificaban si sus filiales extranjeras eran de nueva creación (greenfield investment) o se habían constituido a través de alianzas (joint venture).

También cabe destacar que, para cada cooperativa, sólo se ha considerado la modalidad que implica un mayor grado de internacionalización. Ello se debe a que, siguiendo la lógica secuencial del proceso de internacionalización, es muy probable que una empresa que utilice acuerdos accionariales realice, además, exportaciones y/o tenga acuerdos contractuales. En cambio, si una empresa sólo realiza exportaciones, no estará presente en los mercados internacionales a través de otras modalidades. De esta forma, conseguimos obtener una diferenciación concisa entre los diferentes grados de internacionalización alcanzados por las cooperativas de la muestra.

\section{Resultados y discusión}

En este apartado se presentan y discuten los resultados del análisis sobre las 300 mayores cooperativas del mundo. En primer lugar, se proporciona una imagen general sobre la distribución geográfica y sectorial de estas cooperativas. En los siguientes epígrafes se presentan los análisis específicos diferenciando entre cooperativas internacionalizadas y no internacionalizadas a nivel de facturación, empleo, región geográfica de origen y sector de actividad.

\subsection{Distribución geográfica y sectorial de las 300 mayores cooperativas del mundo}

Como se puede observar en la Tabla 1, las 300 mayores cooperativas del mundo tienen una notable presencia en los principales sectores de actividad que configuran la economía actual. En concreto, buena parte de estas cooperativas desarrollan su actividad en el sector Seguros (alrededor del 40\%), Agricultura y alimentación (en torno al 25\%) y Comercio mayorista y minorista (alrededor del 20\%); sectores donde tradicionalmente el movimiento cooperativo ha tenido una mayor presencia (Monzón 2012). 


\section{Tabla 1. Distribución sectorial de las cooperativas}

\begin{tabular}{|l|cc|cc|cc|cc|}
\hline & \multicolumn{2}{|c|}{$\mathbf{2 0 1 0}$} & \multicolumn{2}{c|}{$\mathbf{2 0 1 1}$} & \multicolumn{2}{c|}{$\mathbf{2 0 1 2}$} & \multicolumn{2}{c|}{$\mathbf{2 0 1 3}$} \\
& Absoluto & Porcentaje & Absoluto & Porcentaje & Absoluto & Porcentaje & Absoluto Porcentaje \\
\hline Agricultura y alimentación & 71 & $23,67 \%$ & 81 & $27,00 \%$ & 80 & $26,67 \%$ & 91 & $30,33 \%$ \\
Servicios bancarios y financieros & 15 & $5,00 \%$ & 14 & $4,67 \%$ & 13 & $4,33 \%$ & 14 & $4,67 \%$ \\
Asistencia sanitaria y social & 5 & $1,67 \%$ & 4 & $1,33 \%$ & 3 & $1,00 \%$ & 5 & $1,67 \%$ \\
Industria & 16 & $5,33 \%$ & 15 & $5,00 \%$ & 16 & $5,33 \%$ & 13 & $4,33 \%$ \\
Seguros & 125 & $41,67 \%$ & 123 & $41,00 \%$ & 123 & $41,00 \%$ & 121 & $40,33 \%$ \\
Otros servicios & 2 & $0,67 \%$ & 1 & $0,33 \%$ & 4 & $1,33 \%$ & 4 & $1,33 \%$ \\
Comercio mayorista y minorista & 66 & $22,00 \%$ & 62 & $20,67 \%$ & 61 & $20,33 \%$ & 52 & $17,33 \%$ \\
Total & 300 & $100 \%$ & 300 & $100 \%$ & 300 & $100 \%$ & 300 & $100 \%$ \\
\hline
\end{tabular}

FUENTE: Elaboración propia a partir del World Cooperative Monitor (2012, 2013, 2014, 2015).

Por otra parte, cabe señalar que, en términos absolutos, las 300 mayores cooperativas del mundo provienen fundamentalmente de grandes países como Estados Unidos, Japón, Francia o Alemania. No obstante, entendemos que es más relevante mostrar la importancia relativa de estas cooperativas en cada país. En la tabla 2 se recoge la densidad cooperativa medida en función del número de cooperativas por cada mil millones de habitantes. Como se puede observar, las mayores cooperativas del mundo tienen particular relevancia en países de reducida dimensión y principalmente europeos, tales como Finlandia, Dinamarca, Noruega, Holanda y Bélgica. Estas cooperativas también tienen una notable presencia en países donde el sector cooperativo ha estado arraigado históricamente, como Francia, Italia, España y Alemania.

\subsection{Grado de internacionalización en las 300 mayores cooperativas del mundo}

A continuación analizamos la internacionalización de las 300 mayores cooperativas del mundo. La Tabla 3 presenta los porcentajes representativos de cada modalidad de presencia en los mercados extranjeros. 
Tabla 2. Densidad cooperativa ( $n^{\circ}$ de cooperativas/población en miles de millones)

\begin{tabular}{|lcccc|}
\hline & $\mathbf{2 0 1 0}$ & $\mathbf{2 0 1 1}$ & $\mathbf{2 0 1 2}$ & $\mathbf{2 0 1 3}$ \\
\hline Finlandia & 1,86 & 2,04 & 2,03 & 2,02 \\
Dinamarca & 1,44 & 1,44 & 1,61 & 1,78 \\
Noruega & 1,23 & 1,41 & 1,59 & 1,38 \\
Nueva Zelanda & 1,15 & 1,14 & 1,13 & 1,35 \\
Holanda & 0,60 & 0,84 & 0,90 & 1,07 \\
Bélgica & 0,37 & 0,36 & 0,45 & 0,89 \\
Suiza & 1,28 & 1,26 & 0,88 & 0,86 \\
Irlanda & 0,44 & 0,44 & 0,44 & 0,65 \\
Suecia & 0,64 & 0,63 & 0,63 & 0,62 \\
Francia & 0,65 & 0,62 & 0,65 & 0,60 \\
Austria & 0,36 & 0,36 & 0,36 & 0,59 \\
Alemania & 0,43 & 0,44 & 0,40 & 0,41 \\
Singapur & 0,39 & 0,39 & 0,57 & 0,37 \\
Canadá & 0,29 & 0,29 & 0,29 & 0,28 \\
Estados Unidos & 0,26 & 0,26 & 0,27 & 0,23 \\
Italia & 0,35 & 0,22 & 0,22 & 0,22 \\
España & 0,17 & 0,17 & 0,19 & 0,15 \\
Japón & 0,15 & 0,15 & 0,13 & 0,14 \\
Australia & 0,14 & 0,13 & 0,13 & 0,13 \\
Reino Unido & 0,16 & 0,16 & 0,14 & 0,11 \\
República de Corea & $\cdot$ & $\cdot$ &. & 0,08 \\
Arabia Saudí &. &. & 0,03 & 0,03 \\
Brasil & 0,02 & 0,03 & 0,01 & 0,03 \\
Colombia & 0,02 &. &. & 0,02 \\
Corea del Sur & 0,02 & 0,04 & 0,04 & 0,02 \\
India & 0,00 & 0,00 & 0,00 & 0,00 \\
República Checa & $\cdot$ & $\cdot$ & 0,10 &. \\
Argentina & $\cdot$ & 0,02 &. \\
\hline
\end{tabular}

FUENTE: Elaboración propia a partir del World Cooperative Monitor $(2012,2013,2014,2015)$ y de las bases de datos de la OCDE y del Banco Mundial.

Tabla 3. Grado de internacionalización de las cooperativas

\begin{tabular}{|l|cccc|}
\hline & $\mathbf{2 0 1 0}$ & $\mathbf{2 0 1 1}$ & $\mathbf{2 0 1 2}$ & $\mathbf{2 0 1 3}$ \\
\hline Total de cooperativas internacionalizadas & $83,09 \%$ & $85,12 \%$ & $85,58 \%$ & $85,78 \%$ \\
Acuerdos accionariales & $55,07 \%$ & $54,42 \%$ & $55,35 \%$ & $54,59 \%$ \\
Acuerdos contractuales & $19,81 \%$ & $20,00 \%$ & $21,40 \%$ & $20,64 \%$ \\
Exportación & $8,21 \%$ & $10,70 \%$ & $8,84 \%$ & $10,55 \%$ \\
No internacionalización & $16,91 \%$ & $14,88 \%$ & $14,42 \%$ & $14,22 \%$ \\
\hline
\end{tabular}

FUENTE: Elaboración propia a partir del World Cooperative Monitor $(2012,2013,2014,2015)$ y de las memorias anuales de las cooperativas. 
Como se puede observar, en todos los años entre 2010 y 2013, alrededor del $85 \%$ de las 300 mayores cooperativas del mundo desarrollan su actividad en los mercados extranjeros, bien mediante exportaciones, acuerdos contractuales o acuerdos accionariales. De esta forma, podemos señalar que estas cooperativas tienen una sólida presencia internacional. En concreto, cerca del $55 \%$ de las cooperativas están presentes internacionalmente a través de acuerdos accionariales. Ello pone de relieve el elevado grado de internacionalización de las 300 mayores cooperativas del mundo, ya que esta modalidad es la que conlleva un mayor nivel de implicación internacional, tanto a nivel de recursos comprometidos y riesgo, como de posibles rendimientos. Asimismo, alrededor del $20 \%$ de las cooperativas están presentes en los mercados extranjeros a través de acuerdos contractuales, que suponen un grado de compromiso internacional algo menor. Por otro lado, cerca del $10 \%$ de las cooperativas llevan a cabo únicamente exportaciones, que se erige como la modalidad de menor implicación internacional. Finalmente, observamos que una muestra estable de alrededor del $15 \%$ de las cooperativas desarrolla su actividad exclusivamente en las fronteras nacionales.

\subsection{Tamaño de las cooperativas en términos de facturación y empleo}

A continuación, se realiza un análisis comparativo sobre la facturación y empleo de las cooperativas en función de la modalidad de presencia en los mercados internacionales. La Tabla 4 muestra las cifras absolutas de facturación (expresado en millones de dólares americanos) y empleo (expresado en número de trabajadores). Cabe recordar que las cifras de empleo incluyen el $49,67 \%$ del total de cooperativas en el año 2010; el 47,67\% en 2011; el 48,67\% en 2012; y el 52\% en 2013.

Un primer aspecto destacable es que el conjunto de cooperativas internacionales (incluyendo las tres modalidades de internacionalización) alcanzó en 2010 una facturación cercana a los 1.500 millones de dólares y empleó directamente a más de 2 millones de trabajadores, aumentando hasta alrededor de 1.900 millones de dólares y 2,3 millones de trabajadores en el año 2013 (considerando que sólo se dispone de datos de empleo para alrededor del 50\% de las cooperativas en cada uno de los años). Las cooperativas presentes en los mercados extranjeros a través de acuerdos accionariales son las que presentan mayores niveles de facturación y empleo en términos relativos. 
Tabla 4. Facturación y empleo de las cooperativas

\begin{tabular}{|c|c|c|c|c|}
\hline & \multicolumn{2}{|c|}{2010} & \multicolumn{2}{|c|}{2011} \\
\hline & Facturación & Empleo & Facturación & Empleo \\
\hline Total de cooperativas internacionalizadas & $1.499,69$ & 2.008 .879 & $1.655,17$ & 2.164 .805 \\
\hline Acuerdos accionariales & $1.252,92$ & 1.879 .060 & $1.349,11$ & 2.034 .509 \\
\hline Acuerdos contractuales & 199,05 & 63.526 & 240,10 & 71.862 \\
\hline Exportación & 47,72 & 66.293 & 65,96 & 58.434 \\
\hline \multirow[t]{3}{*}{ No internacionalizadas } & 189,45 & 367.186 & 184,59 & 325.170 \\
\hline & \multicolumn{2}{|c|}{2012} & \multicolumn{2}{|c|}{2013} \\
\hline & Facturación & Empleo & Facturación & Empleo \\
\hline Total de cooperativas internacionalizadas & $1.759,62$ & 2.195 .490 & $1.859,32$ & 2.322 .458 \\
\hline Acuerdos accionariales & $1.418,71$ & 2.061 .609 & $1.473,46$ & 2.103 .420 \\
\hline Acuerdos contractuales & 282,22 & 102.851 & 299,13 & 174.136 \\
\hline Exportación & 58,69 & 31.030 & 86,73 & 44.902 \\
\hline No internacionalizadas & 185,41 & 324.264 & 211,57 & 312.848 \\
\hline
\end{tabular}

FUENTE: Elaboración propia a partir del World Cooperative Monitor $(2012,2013,2014,2015)$ y de las memorias anuales de las cooperativas.

Comparativamente, el conjunto de cooperativas internacionalizadas ha experimentado una evolución significativamente mejor, tanto a nivel de facturación como de empleo, que las cooperativas no internacionalizadas durante el periodo 2010-2013, caracterizado por una compleja coyuntura de recesión económica. El conjunto de cooperativas internacionalizadas han aumentado su facturación un $10,37 \%$ entre 2010 y 2011 ; un 6,31\% entre 2011 y 2012; y un 5,67\% entre 2012 y 2013 . Por el contrario, la facturación de las cooperativas no internacionalizadas cayó un 2,57\% entre 2010 y 2011, y sólo aumentó un 0,44\% el siguiente año. En cambio, el incremento en 2013 es mayor en las cooperativas no internacionalizadas $(14,11 \%)$ que en las internacionalizadas $(5,67 \%)$. La tendencia señalada es todavía más evidente en la evolución del empleo. Mientras las cooperativas internacionalizadas muestran incrementos anuales del 7,76\%, 1,42\% y 5,78\% en 2011, 2012 y 2013, las cooperativas no internacionalizadas presentan tasas de destrucción de empleo del $11,44 \%, 0,28 \%$ y $3,52 \%$, respectivamente.

Estos resultados son consistentes con la literatura que ha analizado la respuesta comparativa de las empresas internacionalizadas y domésticas en términos de facturación y empleo ante coyunturas de recesión económica. En este sentido, un amplio número de trabajos han demostrado que las empresas internacionalizadas resisten situaciones de crisis económica en mejores condiciones que las empresas que operan exclusivamente a nivel doméstico, siendo capaces de mantener mejor los niveles de empleo y de facturación, debido a la flexibilidad que otorga el hecho de operar en diver- 
sos mercados extranjeros (Lee y Makhija, 2009; Varum y Rocha, 2011). Estos estudios destacan diversos motivos. Entre ellas, las empresas internacionalizadas cuentan con ventajas financieras, de conocimientos y tecnológicas que les dotan de capacidades superiores para competir y hacer frente a cambios en las condiciones del mercado. Asimismo, las empresas que cuentan con filiales en diversos países dispondrán de flexibilidad para reubicar la producción ante coyunturas negativas en un determinado país, como las producidas por una crisis económica. Además, las empresas que disponen de una infraestructura sólida de exportación pueden hacer frente a caídas drásticas de la demanda interna trasladando sus ventas a mercados más rentables.

\subsection{Región de origen y sector de actividad de las cooperativas}

Debido a que las 300 mayores cooperativas del mundo tienen su lugar de origen en 27 países diferentes, se ha analizado su localización atendiendo a la región de procedencia, distinguiendo entre Europa, América del Norte y Asia (Tabla 5). Se han excluido América del Sur y Oceanía debido a que el reducido número de cooperativas hace que estas regiones no sean representativas. De hecho las cooperativas procedentes de Europa, América del Norte y Asia representan una muestra superior al 95\% de cooperativas en todos los años del periodo 2010-2013.

Es reseñable que no existen grandes diferencias en el grado de internacionalización de las cooperativas dependiendo de su región geográfica de origen. Como se observa, alrededor del $80-85 \%$ de las cooperativas procedentes de Europa, América del Norte y Asia están presentes en los mercados internacionales. En cambio, sí que se observan diferencias en los modos de presencia internacional de las cooperativas provenientes de las diferentes regiones geográficas. En el caso de las cooperativas europeas, y especialmente las asiáticas, existe una mayor tendencia a adoptar acuerdos accionariales en comparación con las norteamericanas, las cuales son más propensas a expandirse internacionalmente mediante la exportación y acuerdos contractuales. 


\section{Tabla 5. Porcentaje de cooperativas según modalidad de presencia internacional y región geográfica de origen}

\begin{tabular}{|c|c|c|c|c|c|c|c|c|}
\hline & \multicolumn{4}{|c|}{ Europa } & \multicolumn{4}{|c|}{ América del Norte } \\
\hline & 2010 & 2011 & 2012 & 2013 & 2010 & 2011 & 2012 & 2013 \\
\hline Total cooperativas internacionalizadas & $82,93 \%$ & $84,92 \%$ & $87,12 \%$ & $86,67 \%$ & $81,25 \%$ & $84,85 \%$ & $81,25 \%$ & $83,33 \%$ \\
\hline Acuerdos accionariales & $60,98 \%$ & $61,90 \%$ & $62,12 \%$ & $61,48 \%$ & $42,19 \%$ & $40,91 \%$ & $37,50 \%$ & $40,00 \%$ \\
\hline Acuerdos contractuales & $17,07 \%$ & $17,46 \%$ & $18,94 \%$ & $18,52 \%$ & $29,69 \%$ & $30,30 \%$ & $31,25 \%$ & $31,67 \%$ \\
\hline Exportación & $4,88 \%$ & $5,56 \%$ & $6,06 \%$ & $6,67 \%$ & $9,38 \%$ & $13,64 \%$ & $12,50 \%$ & $11,67 \%$ \\
\hline \multirow[t]{3}{*}{ Nacional } & $17,07 \%$ & $15,08 \%$ & $12,88 \%$ & $13,33 \%$ & $18,75 \%$ & $15,15 \%$ & $18,75 \%$ & $16,67 \%$ \\
\hline & \multicolumn{4}{|c|}{ Asia } & & & & \\
\hline & 2010 & 2011 & 2012 & 2013 & & & & \\
\hline Total cooperativas internacionalizadas & $80,00 \%$ & $72,73 \%$ & $81,82 \%$ & $75,00 \%$ & & & & \\
\hline Acuerdos accionariales & $70,00 \%$ & $63,64 \%$ & $72,73 \%$ & $58,33 \%$ & & & & \\
\hline Acuerdos contractuales & $10,00 \%$ & $9,09 \%$ & $9,09 \%$ & $8,33 \%$ & & & & \\
\hline Exportación & $0,00 \%$ & $0,00 \%$ & $0,00 \%$ & $8,33 \%$ & & & & \\
\hline Nacional & $20,00 \%$ & $27,27 \%$ & $18,18 \%$ & $25,00 \%$ & & & & \\
\hline
\end{tabular}

FUENTE: Elaboración propia a partir del World Cooperative Monitor $(2012,2013,2014,2015)$ y de las memorias anuales de las cooperativas.

Estos resultados podrían encontrar relación con la literatura que ha analizado la vinculación entre la distancia cultural y el modo de entrada en los mercados extranjeros (véase Brouthers y Hennart 2007 para una revisión). En términos generales, se señala que si existe una gran distancia cultural entre el país de origen de la empresa y el país extranjero al que accede, la empresa preferirá utilizar modalidades de menor riesgo como los acuerdos contractuales o joint ventures (Hennart y Larimo, 1998). No obstante, nuestros resultados no permiten obtener conclusiones en esta dirección. Asimismo, existe consenso en la literatura en que el sector de actividad es más relevante para explicar las diferencias en los modos de entrada (Tihanyi et al., 2005). A partir de la Tabla 6, analizamos la relación entre el sector de las cooperativas y el modo de internacionalización. Los sectores "Asistencia sanitaria y social" y "Otros servicios" se han excluido al representar una parte residual del conjunto de cooperativas.

En primer lugar, cerca del $97 \%$ de las cooperativas del sector "Agricultura y alimentación" desarrolla su actividad en el extranjero mediante exportaciones, acuerdos contractuales y accionariales. Esto es consistente con diversos estudios que han documentado el intenso proceso de globalización del sector agroalimentario, potenciado por las reformas orientadas a desmantelar las barreras comerciales, eliminar los subsidios a la producción, y promover el libre mercado de productos agroalimentarios (por ejemplo, Cheshire y Woods, 2013). Asimismo, la modalidad de exportación tiene una mayor presencia en este sector en comparación con otros. Como señalan Fernández-Olmos y Díez-Vial 
(2014), la exportación sigue siendo una de las principales estrategias de internacionalización en el sector agroalimentario debido, por un lado, a factores como los continuos avances tecnológicos en el transporte y conservación de los alimentos, la caída de la demanda interna y el aumento de la oferta, la disminución del apoyo público y la entrada de nuevos países productores con estrategias comerciales y de producción innovadoras y, por otro lado, a que esta estrategia permite acceder a mercados extranjeros a un coste mucho menor y con elevados niveles de flexibilidad.

Por su parte, cerca del $90 \%$ de cooperativas del sector "Servicios bancarios y financieros" desarrollan su actividad más allá de las fronteras nacionales, mostrando además un elevado grado de internacionalización, ya que la mayor parte de las cooperativas internacionalizadas están presentes en el extranjero mediante acuerdos accionariales. En este sentido, trabajos como el de Petrou (2009) evidencian que los acuerdos accionariales (principalmente las joint-ventures y las adquisiciones) son los modos de entrada dominantes en el sector bancario debido a diversas razones como la saturación del sector en los mercados nacionales y la tendencia hacia una mayor concentración de la industria (favoreciendo la proliferación de acuerdos accionariales); la necesidad de establecer joint-ventures debido a la elevada regulación del sector bancario en los países y a la importancia que tienen los recursos locales en dicho sector (como la distribución, imagen local, conocimiento de mercado y base de clientes); $y$ el hecho de que el sector bancario es un sector intensivo en información donde se genera un gran valor añadido cuando la información es compartida de manera adecuada y rápida a lo largo de la red transnacional, lo que lleva a las empresas a preferir acuerdos accionariales al permitir un mayor control sobre sus negocios internacionales.

Por otra parte, es relevante atender a la diferenciación en los modos de entrada entre el sector industrial manufacturero y el sector servicios. Frente a las empresas manufactureras, las empresas de servicios tienden a utilizar modos de entrada que implican un elevado grado de control (como greenfield investments o joint-ventures mayoritarias) en mayor medida que la exportación o los acuerdos contractuales (Blomstermo et al. 2006; Bouquet et al. 2004; Brouthers y Brouthers, 2003). Estos estudios destacan varios motivos. En primer lugar, las empresas manufactureras suelen requerir una mayor cantidad de recursos para implantarse en el exterior (adquisición/apertura de la planta, instalaciones, equipamiento, etc.), mientras que el compromiso de recursos es menor en las empresas de servicios, pudiendo así acceder a los mercados extranjeros a través de estrategias de elevado control a un coste mucho menor. Otro aspecto clave es que las empresas de servicios dependen habitualmente en mayor medida de activos de mayor especificidad (capital humano, habilidades profesionales, know-how especializado), lo que puede conllevar la preferencia de modos de entrada de mayor integración (greenfield investments o joint-ventures mayoritarias) debido a que los beneficios de estas alternativas pueden ser mayores independientemente de los recursos comprometidos. Por último, la localización es generalmente más importante en las empresas de servicios que en las empresas manufactureras, ya que en las primeras existe una mayor necesidad de acceder a los mercados internacionales con rapidez, de situarse en diversas localizaciones exteriores, y de adaptarse en mayor medida al entorno local; aspectos que pueden lograrse de manera más eficiente a través de acuerdos accionariales. 


\section{Tabla 6. Porcentaje de cooperativas según modalidad de presencia internacional y sector de actividad}

\begin{tabular}{|c|c|c|c|c|c|c|c|c|}
\hline & \multicolumn{4}{|c|}{ Agricultura y alimentación } & \multicolumn{4}{|c|}{ Servicios bancarios y financieros } \\
\hline & 2010 & 2011 & 2012 & 2013 & 2010 & 2011 & 2012 & 2013 \\
\hline Total cooperativas internacionalizadas & $96,88 \%$ & $97,18 \%$ & $97,06 \%$ & $97,40 \%$ & $90,00 \%$ & $90,91 \%$ & $90,00 \%$ & $90,00 \%$ \\
\hline Acuerdos accionariales & $64,06 \%$ & $59,15 \%$ & $66,18 \%$ & $63,64 \%$ & $90,00 \%$ & $81,82 \%$ & $90,00 \%$ & $80,00 \%$ \\
\hline Acuerdos contractuales & $12,50 \%$ & $11,27 \%$ & $11,76 \%$ & $10,39 \%$ & $0,00 \%$ & $9,09 \%$ & $0,00 \%$ & $10,00 \%$ \\
\hline Exportación & $20,31 \%$ & $26,76 \%$ & $19,12 \%$ & $23,38 \%$ & $0,00 \%$ & $0,00 \%$ & $0,00 \%$ & $0,00 \%$ \\
\hline \multirow[t]{3}{*}{ Nacional } & $3,13 \%$ & $2,82 \%$ & $2,94 \%$ & $2,60 \%$ & $10,00 \%$ & $9,09 \%$ & $10,00 \%$ & $10,00 \%$ \\
\hline & \multicolumn{4}{|c|}{ Industria } & \multicolumn{4}{|c|}{ Seguros } \\
\hline & 2010 & 2011 & 2012 & 2013 & 2010 & 2011 & 2012 & 2013 \\
\hline Total cooperativas internacionalizadas & $53,85 \%$ & $58,33 \%$ & $57,14 \%$ & $60,00 \%$ & $89,02 \%$ & $88,89 \%$ & $91,25 \%$ & $91,14 \%$ \\
\hline Acuerdos accionariales & $46,15 \%$ & $50,00 \%$ & $50,00 \%$ & $50,00 \%$ & $57,32 \%$ & $58,02 \%$ & $56,25 \%$ & $55,70 \%$ \\
\hline Acuerdos contractuales & $7,69 \%$ & $8,33 \%$ & $7,14 \%$ & $10,00 \%$ & $31,71 \%$ & $30,86 \%$ & $35,00 \%$ & $35,44 \%$ \\
\hline Exportación & $0,00 \%$ & $0,00 \%$ & $0,00 \%$ & $0,00 \%$ & $0,00 \%$ & $0,00 \%$ & $0,00 \%$ & $0,00 \%$ \\
\hline \multirow[t]{3}{*}{ Nacional } & $46,15 \%$ & $41,67 \%$ & $42,86 \%$ & $40,00 \%$ & $10,98 \%$ & $11,11 \%$ & $8,75 \%$ & $8,86 \%$ \\
\hline & \multicolumn{4}{|c|}{ Comercio mayorista y minorista } & & & & \\
\hline & 2010 & 2011 & 2012 & 2013 & & & & \\
\hline Total cooperativas internacionalizadas & $58,33 \%$ & $64,10 \%$ & $65,00 \%$ & $60,53 \%$ & & & & \\
\hline Acuerdos accionariales & $30,56 \%$ & $33,33 \%$ & $27,50 \%$ & $28,95 \%$ & & & & \\
\hline Acuerdos contractuales & $16,67 \%$ & $20,51 \%$ & $22,50 \%$ & $18,42 \%$ & & & & \\
\hline Exportación & $11,11 \%$ & $10,26 \%$ & $15,00 \%$ & $13,16 \%$ & & & & \\
\hline Nacional & $41,67 \%$ & $35,90 \%$ & $35,00 \%$ & $39,47 \%$ & & & & \\
\hline
\end{tabular}

FUENTE: Elaboración propia a partir del World Cooperative Monitor $(2012,2013,2014,2015)$ y de las memorias anuales de las cooperativas.

No obstante, nuestros resultados plantean matizaciones importantes. En primer lugar, es reseñable que el sector "Industria" aglutina la mayor proporción de cooperativas no internacionalizadas. Una explicación plausible es que las cooperativas industriales son propiedad de los propios trabajadores, quienes toman las decisiones sobre la empresa de manera directa. Por tanto, los incentivos a expandirse internacionalmente en este tipo de cooperativas pueden ser menores debido a que los socios-trabajadores tienden a proteger su inversión en la cooperativa - la expansión internacional podría implicar la pérdida del control sobre el capital - y a otros factores como el deseo de preservar los mecanismos democráticos en la gestión de la empresa y de mantener la toma de decisiones por parte de los socios-trabajadores anclada al territorio local (Bretos et al. 2018a, 2018b). Asimismo, se observa que las cooperativas de este sector están presentes en los mercados extranjeros a través de acuerdos accionariales fundamentalmente. En este sentido, podemos postular que, en el caso de inter- 
nacionalizarse, las cooperativas industriales preferirán modos de entrada internacional que impliquen un elevado grado de control, de manera que los socios-trabajadores mantengan su independencia y control sobre la toma de decisiones de la empresa.

Con respecto al sector "Seguros", se observa un elevado nivel de internacionalización de sus cooperativas (alrededor del 90\% están presentes en mercados extranjeros). En la línea de lo que señala Birchall (2014), esto es lógico si tenemos en cuenta que en las cooperativas o mutuas de seguros, los miembros tienen un incentivo para animar a sus gerentes a crecer indefinidamente, ya que cuantas más personas estén aseguradas, mayor es la diversificación del riesgo y más barato es el coste. Asimismo, a diferencia de otras cooperativas como las de consumidores, donde existe una sólida relación económico-social entre miembro y cooperativa, las cooperativas de seguros pueden crecer más fácilmente ya que la conexión miembro-cooperativa no se extiende habitualmente más allá de una prima anual.

Los resultados sobre el sector "Seguros" parecen cumplir lo señalado por la literatura en relación a los modos de entrada en el sector servicios, ya que los acuerdos accionariales es la modalidad mayoritaria en este sector (cerca del $60 \%$ del total). Sin embargo, también se observa una importancia significativa de los acuerdos contractuales (alrededor del $30 \%$ ). En este sentido, gana relevancia la distinción entre los denominados "soft services" y "hard services" (Bouquet et al., 2004; Brouthers y Brouthers 2003). Los primeros son aquellos en que la producción y el consumo tienen lugar simultáneamente y, por tanto, no son fácilmente separables. Ello implica el posible requerimiento de implantarse en el exterior a través de acuerdos accionariales debido a la necesidad de proximidad física entre el proveedor y el consumidor. En cambio, los segundos son aquellos servicios en que la producción y el consumo son separables (como en el sector "Insurance"), siendo más fácil desarrollar estrategias de internacionalización que implican un reducido control (Bouquet et al., 2004).

En relación al sector "Comercio mayorista y minorista", destaca en primer lugar la importante relevancia de las cooperativas no internacionalizadas (alrededor del $35-40 \%$ de las cooperativas). Una posible explicación es que las cooperativas de consumo pueden tener menores incentivos para crecer internacionalmente por motivos como el sólido vínculo territorial de los socios a las necesidades surgidas en el ámbito local o regional, o las dificultades para alinear los intereses individuales y colectivos al expandirse internacionalmente, en tanto que puede dificultar la fidelidad de los miembros consumidores y la capacidad de respuesta de la cooperativa a sus necesidades (Birchall, 2014). Por otro lado, se observa una distribución relativamente homogénea de las diferentes modalidades ( $30 \%$ acuerdos accionariales, $20 \%$ acuerdos contractuales y $10 \%$ exportaciones). Ello puede deberse a la heterogeneidad en las actividades económicas que llevan a cabo las cooperativas de este sector. Como señalan diversos trabajos (entre otros, Blomstermo et al. 2006; Bouquet et al. 2004), las operaciones de comercio minorista tienen lugar simultáneamente con el consumo en una interacción cercana con el consumidor y, por tanto, producción y consumo no son fácilmente separables (soft service). Por ello, las cooperativas de comercio minorista pueden requerir de acuerdos accionariales para implantarse en el exterior. En cambio, las empresas de comercio mayorista suelen ofrecer servicios como aseso- 
ramiento técnico, logístico y financiero, o servicios de marketing, los cuales pueden llevarse a cabo aisladamente, es decir, en ausencia de una interacción cercana entre proveedor y consumidor, existiendo por tanto una mayor separabilidad entre producción y consumo (hard service). De esta forma, las cooperativas de comercio mayorista pueden desarrollar más fácilmente estrategias de exportación y acuerdos contractuales.

\section{Conclusiones}

Mediante el análisis de las 300 mayores cooperativas del mundo en el periodo 2010-2013, este artículo ha contrastado el grado de internacionalización de estas cooperativas, las regiones geográficas donde están localizadas, los sectores económicos donde desarrollan su actividad y su tamaño en términos de facturación y empleo. Los datos se han obtenido principalmente del World Cooperative Monitor elaborado por la Alianza Cooperativa Internacional y el Instituto Euricse y de la propia información divulgada públicamente por las cooperativas en sus informes anuales.

A partir de la revisión de la literatura sobre la internacionalización empresarial y sobre las características de índole económica y organizacional de las cooperativas, hemos identificado 5 factores clave que sugieren que estas organizaciones deberían ser incapaces de expandirse más allá de sus fronteras nacionales y sobrevivir en entornos de competencia internacional: (1) la creación limitada de cooperativas y la dimensión marginal del sector cooperativo en la mayoría de países y sectores; (2) la reticencia e incapacidad de las cooperativas para crecer; (3) las dificultades de las cooperativas para atraer y retener directivos capacitados y experimentados; (4) los mecanismos ineficientes de las cooperativas en la toma de decisiones; y (5) la limitada capacidad financiera y de inversión en las cooperativas.

En cambio, nuestros resultados cuestionan estas predicciones. Por un lado, del análisis de los datos se deduce que existen cooperativas de gran tamaño en los principales sectores de actividad y economías mundiales. Estas grandes cooperativas tienen especial incidencia en pequeñas economías europeas avanzadas como Finlandia, Dinamarca, Noruega, Holanda y Bélgica, aunque también son destacables otros países de mayor tamaño donde el sector cooperativo tiene una larga tradición, como Francia, Alemania, España e Italia. A nivel sectorial, las mayores cooperativas del mundo desarrollan su actividad fundamentalmente en los sectores de Seguros, Agricultura y alimentación, Comercio mayorista y minorista, Servicios bancarios y financieros, e Industria.

Por otro lado, nuestros resultados sugieren que la internacionalización dista mucho de ser un fenómeno marginal en las cooperativas. Una muestra estable de alrededor del $85 \%$ de las cooperativas 
desarrolla su actividad más allá de sus fronteras nacionales en todos los años del periodo 2010-2013. Además, destaca su elevado grado de internacionalización. El 55\% de las cooperativas están presentes en los mercados extranjeros a través de acuerdos accionariales (que implican el mayor nivel de compromiso internacional en términos de inversión, riesgo, rendimiento y control); el $20 \%$ a través de acuerdos accionariales (nivel intermedio de compromiso internacional); y el 10\% a través de exportaciones (menor nivel de compromiso internacional).

En cuanto a la forma de internacionalización, son relevantes las variaciones detectadas de acuerdo al sector de actividad. La práctica totalidad de las cooperativas del sector "Agricultura y alimentación" operan a nivel internacional, siendo particularmente relevante la estrategia de exportación en comparación con otros sectores. Asimismo, alrededor del $90 \%$ de las cooperativas de los sectores "Servicios bancarios y financieros" y "Seguros" están presentes en los mercados internacionales. No obstante, las primeras muestran un grado de internacionalización mayor, debido al elevado uso de acuerdos accionariales, en contraposición con las segundas que utilizan en mayor medida los acuerdos contractuales para acceder al extranjero. Por su parte, los sectores "Industria" y "Comercio mayorista y minorista" aglutinan el mayor número de cooperativas que operan exclusivamente a nivel doméstico (alrededor del $40 \%$ de cooperativas). Además, existen diferencias sustanciales en el grado de compromiso internacional entre ambos sectores. Mientras que las cooperativas internacionalizadas del sector "Industria" están presentes en los mercados internacionales mayoritariamente a través de acuerdos accionariales, el sector "Comercio mayorista y minorista" destaca por la heterogeneidad de las diferentes estrategias de internacionalización empleadas. Estas variaciones parecen atender a factores diversos, como la estructura sectorial, la naturaleza de las actividades económicas en función de su "transferibilidad" a los mercados extranjeros, o las características organizacionales y preferencias/necesidades de los socios de las distintas tipologías de cooperativas que predominan en cada sector.

Asimismo, nuestros resultados complementan investigaciones previas acerca del comportamiento de las cooperativas ante coyunturas de recesión económica a nivel regional (Bretos y Morandeira 2016) y nacional (Pérotin 2006), mostrando que las 300 mayores cooperativas del mundo, provenientes de una muestra heterogénea de sectores y países, han sido capaces de aumentar sus niveles de facturación y empleo en un periodo 2010-2013 caracterizado por una fuerte crisis económica global. Además, se observa que las cooperativas internacionalizadas muestran una mejor evolución durante dicho periodo en comparación con las cooperativas exclusivamente domésticas tanto a nivel de empleo como de facturación. Estos resultados parecen confirmar algunas conclusiones de la literatura previa que demuestran que las empresas que operan en los mercados extranjeros cuentan con mejores capacidades y mayor flexibilidad para resistir las situaciones de crisis económica (Lee y Makhija, 2009; Varum y Rocha, 2011). En particular, el conjunto de cooperativas internacionalizadas alcanzó en 2010 una facturación cercana a los 1.500 millones de dólares y empleó directamente a más de 2 millones de trabajadores, aumentando hasta alrededor de 1.900 millones de dólares y 2,3 millones de empleos en el año 2013. 
En conclusión, esta investigación desafía diversos argumentos teóricos que apuntan a la ineficiencia de las cooperativas y su incapacidad para crecer en un contexto global, los cuales han impregnado el imaginario colectivo y siguen muy presentes en la literatura científica. En este sentido, contribuimos a recientes estudios que han advertido la necesidad de revisar nuestra comprensión sobre estas organizaciones (Pérotin 2015; Bretos y Marcuello), demostrando que existen grandes cooperativas internacionalizadas que compiten de manera eficaz en los principales sectores económicos y países.

\subsection{Limitaciones y líneas de investigación futura}

La naturaleza exploratoria de esta investigación conlleva diversas limitaciones, a la vez que también abre importantes líneas para la investigación futura. En primer lugar, el reducido tamaño de la muestra impide proporcionar una imagen más precisa y detallada sobre la escala del fenómeno de la internacionalización en las cooperativas. Asimismo, el hecho de que la base de datos esté conformada por las mayores cooperativas del mundo conlleva seguramente un sobredimensionamiento de la proporción de cooperativas internacionalizadas. Dichas limitaciones provienen en última instancia de la escasez de estadísticas fiables y precisas sobre el sector cooperativo a nivel internacional (Monzón 2012).

Por otro lado, en este artículo hemos planteado una serie de hipótesis conectando la literatura sobre las cooperativas con la literatura sobre la internacionalización, que sugieren que estas organizaciones adolecen de serias limitaciones para crecer internacionalmente. Si bien nuestra investigación demuestra que existen cooperativas internacionalizadas en las principales economías mundiales y sectores de actividad, nuestros datos sólo nos han permitido contrastar estas hipótesis de forma exploratoria. Por tanto, todavía queda mucho por conocer acerca de cómo las cooperativas se internacionalizan. Por ejemplo, podríamos preguntarnos cómo estas cooperativas han hecho frente a sus limitaciones para atraer y retener directivos valiosos o para disponer de capacidad de inversión. Teniendo en cuenta la limitada disponibilidad de datos a gran escala en este ámbito, la investigación futura podría ahondar en estas cuestiones a través de estudios cualitativos, debido a su idoneidad para promover nuestro conocimiento en áreas donde la investigación previa es limitada.

Otra cuestión clave consiste en refinar nuestro conocimiento sobre las estrategias de internacionalización empleadas por las cooperativas. Entre otras cuestiones, nuestra investigación no ha podido identificar las distintas tipologías de acuerdos accionariales elegidas por las cooperativas (por ejemplo, greenfield investment frente a joint venture), a pesar de que existen importantes diferencias entre ambas estrategias en términos del grado de internacionalización. Por tanto, avanzar en esta línea permitiría obtener un conocimiento más preciso sobre el grado de internacionalización de las cooperativas. Asimismo, hemos observado que existen diferencias significativas entre las estrategias de internacionalización empleadas por las cooperativas en función del sector de actividad. Si bien hemos tratado de proporcionar algunas explicaciones tentativas basadas en la literatura previa, no cabe duda de que es necesario seguir progresando en esta dirección para conocer los factores que llevan a las cooperativas a utilizar una u otra estrategia de expansión internacional. 
Finalmente, en la línea de algunos estudios recientes (entre otros, Flecha y Ngai 2014; Errasti et al. 2017; Bretos et al. 2018a, 2018b), también resulta esencial continuar avanzando en nuestra comprensión sobre el comportamiento organizacional de las cooperativas multinacionales. Por ejemplo, ¿siguen las cooperativas patrones de crecimiento internacional más sostenibles en comparación con las empresas capitalistas? ¿Cómo responden las cooperativas internacionalizadas a los intereses de los diferentes grupos de interés que se ven afectados por su actividad global? ¿Qué políticas y prácticas de gestión de recursos humanos adoptan las cooperativas en sus filiales extranjeras? Asimismo, la investigación futura también podría abordar el impacto de la expansión internacional en las prácticas y valores cooperativos en estas organizaciones, preguntándose, por ejemplo, si el crecimiento internacional conlleva el surgimiento de procesos de degeneración cooperativa y, de ser así, qué estrategias pueden desarrollar las cooperativas para hacer frente a estas tendencias degenerativas.

\section{Referencias bibliográficas}

ACQUAAH, M. (2003): "Corporate management, industry competition and the sustainability of firm abnormal profitability", Journal of Management and Governance, 7: 57-85.

ACS, Z.J., MORCK, R.K. \& YEUNG, B. (2001): "Entrepreneurship, globalisation, and public policy", Journal of International Management, 7: 235-251.

AGARWAL, S. \& RAMASWAMI, S.N. (1992): "Choice of Foreign Market Entry Mode: Impact of Ownership, Location and Internalization Factors", Journal of International Business Studies, 27: 1-27.

ARANDO, S., GAGO, M., PODIVINSKY, J.M. \& STEWART, G. (2012): "Do labour-managed firms benefit from agglomeration?", Journal of Economic Behaviour and Organization, 84: 193-200.

ARANDO, S., PEÑA, I. \& VERHEUL, I. (2009): "Market entry of firms with different legal forms: An empirical test of the influence of institutional factors", International Entrepreneurship Management Journal, 5: 77-95.

BARKEMA, H.G. \& VERMEULEN, F. (1997): "What differences in the cultural backgrounds of partners are detrimental for international joint ventures?", Journal of International Business Studies, 28(4): 845-864.

BEN-NER, A. (1988): "The life cycle of worker-owned firms in market economies: A theoretical analysis", Journal of Economic Behavior \& Organization, 10: 287-31.

BIRCHALL, J. (2014): The Governance of Large Co-operative Businesses, Manchester: Cooperatives UK. 
BLOMSTERMO, A., SHARMA, D.D. \& SALLIS, J. (2006): "Choice of Foreign Market Entry Mode in Service Firms", International Marketing Review, 23(2): 211-229.

BOUQUET, C., HEBERT, L. \& DELIOS, A. (2004): "Foreign expansion in service industries: Separability and human-capital intensity", Journal of Business Research, 77(1): 35-46.

BRETOS, I. \& ERRASTI, A. (2016): "Dinámicas de regeneración en las cooperativas multinacionales de Mondragón: La reproducción del modelo cooperativo en las filiales capitalistas", CIRIEC-España, Revista de Economía Pública, Social y Cooperativa, 86: 5-34.

BRETOS, I. \& MORANDEIRA, J. (2016): "La economía social ante la actual crisis económica en la Comunidad Autónoma del País Vasco", REVESCO, Revista de Estudios Cooperativos, 122: 7-33.

BRETOS, I. \& ERRASTI, A. (2017): "Challenges and Opportunities for the Regeneration of Multinational Worker Cooperatives: Lessons from the Mondragon Corporation-A Case Study of the Fagor Ederlan Group", Organization, 24(2): 154-173.

BRETOS, I. \& MARCUELLO, C. (2017): "Revisiting Globalization Challenges and Opportunities in the Development of Cooperatives", Annals of Public and Cooperative Economics, 88(1): 47-73.

BRETOS, I. \& ERRASTI, A. (2018): "La transmisión de los valores y prácticas organizacionales cooperativas en las filiales extranjeras: el caso de la cooperativa multinacional Fagor Ederlan", REVESCO, Revista de Estudios Cooperativos, in press, DOI: http://dx.doi.org/10.5209/REVE.58395.

BRETOS, I., ERRASTI, A. \& MARCUELLO, C. (2018): "Ownership, governance, and the diffusion of HRM practices in multinational worker cooperatives: Case study evidence from the Mondragon group", Human Resource Management Journal, 28: 76-91.

BRETOS, I., ERRASTI, A. \& MARCUELLO, C. (2018): "Multinationalization of worker cooperatives and the cross-national transfer of employment practices: Evidence from Mondragon multinational cooperatives", ILR Review (forthcoming).

BROUTHERS, K.D. \& BROUTHERS, L.E. (2003): "Why service and manufacturing entry mode choices differ: the influence of transaction cost factors, risk, and trust", Journal of Management Studies, 40(5): 1179-1204.

BROUTHERS, K.D. \& HENNART, J.F. (2007): "Boundaries of the Firm: Insights from International Entry Mode Research", Journal of Management, 33(3): 395-425.

CHADDAD, F.R. \& COOK, M.L. (2004): "The economics of organization structure changes: A U.S. perspective on demutualization", Annals of Public and Cooperative Economics 75: 575-594.

CHAVES, R. \& SAJARDO, A. (2004): "Social Economy Managers: Between Values and Entrenchment", Annals of Public and Cooperative Economics, 75(1): 139-161.

CHESHIRE, L. \& WOODS, M. (2013): "Globally engaged farmers as transnational actors: navigating the landscape of agri-food globalization", Geoforum, 44(1): 232-42. 
CORNFORTH, C. \& THOMAS, H. (1990): "Co-operative Development: Barriers, Support Structures and Cultural Factors", Economic and Industrial Democracy, 11: 451-61.

DÍAZ-FONCEA, M. \& MARCUELLO, C. (2015): "Spatial patterns in new firm formation: Are cooperatives different?", Small Business Economics, 44: 171-187.

DOW, G.K. (2003): Governing the Firm: Workers' Control in Theory and Practice, Cambridge: Cambridge University Press.

ERRASTI, A., BRETOS, I. \& ETXEZARRETA, E. (2016): "What do Mondragon Coopitalist Multinationals Look Like? The Rise and Fall of Fagor Electrodomésticos S. Coop. and its European Subsidiaries", Annals of Public and Cooperative Economics, 87: 433-456.

ERRASTI, A., BRETOS, I. \& NUNEZ, A. (2017): "The Viability of Cooperatives: The Fall of the Mondragon Cooperative Fagor", Review of Radical Political Economics, 49(2): 181-197.

FERNÁNDEZ-OLMOS, M. \& DÍEZ-VIAL, I. (2013): "The direct or indirect exporting decision in agri-food firms", Agribusiness, 30(2): 148-164.

FLECHA, R. \& NGAI, P. (2014): "The challenge for Mondragon: Searching for the co-operative values in times of internationalization", Organization, 21(5): 666-682.

GEORGE, G., WIKLUND, J. \& ZAHRA, S.A. (2005): "Ownership and the Internationalization of the Small Firm", Journal of Management, 31(2): 210-233.

HANSMANN, H. (1996): The Ownership of Enterprise, Cambridge: Harvard Press.

HENNART, J.F. \& LARIMO, J. (1998): "The impact of culture on the strategy of multinational enterprises: Does national origin affect ownership decisions?", Journal of International Business Studies, 29(3): 515-538.

HITT, M.A., TIHANYI, L., MILLER, T., et al. (2006): "International Diversification: Antecedents, Outcomes, and Moderators", Journal of Management, 32(6): 831-67.

ICA (2012): The 2012 World Cooperative Monitor, Brussels: International Co-operative Alliance, Euricse Institute. Disponible en: http://monitor.coop/

ICA (2013): The 2013 World Cooperative Monitor, Brussels: International Co-operative Alliance, Euricse Institute. Disponible en: http://monitor.coop/

ICA (2014): The 2014 World Cooperative Monitor, Brussels: International Co-operative Alliance, Euricse Institute. Disponible en: http://monitor.coop/

ICA (2015): The 2015 World Cooperative Monitor, Brussels: International Co-operative Alliance, Euricse Institute. Disponible en: http://monitor.coop/

JENSEN, M.C. \& MECKLING, W. (1979): "Rights and production functions: An application to labormanaged firms and codetermination", Journal of Business, 52: 469-506. 
JOHANSON, J. \& VAHLNE, J.E. (1977): "The internationalization process of the firm: A model of knowledge development and increasing foreign market commitments", Journal of International Business Studies, 8(1): 23-32.

JOHANSON, J. \& VAHLNE, J.E. (2009): "The Uppsala internationalization process model revisited: From liability of foreignness to liability of outsidership", Journal of International Business Studies, 40(9): 1411-31.

JOYCE, P. \& WOODS, A. (2003): "Managing for Growth: Decision Making, Planning and Making Changes", Journal of Small Business and Enterprise Development, 10(2): 144-152.

KEDIA, B.L., NORDTVEDT, R. \& PÉREZ, L.M. (2002): "International Business Strategies, DecisionMaking Theories, and Leadership Styles: an Integrated Framework", Competitiveness Review, 12(1): 38-52.

LEE, S.H. \& MAKHIJA, M. (2009): "Flexibility in internationalization: is it valuable during an economic crisis?", Strategic Management Journal, 30: 537-555.

LU, J. \& BEAMISH, P. (2001): "The internationalization and performance of SMEs", Strategic Management Journal, 22: 565-586.

LUO, Y. (2005): "How Does Globalization Affect Corporate Governance and Accountability? A perspective from MNEs", Journal of International Management, 11: 19-41.

MONZÓN, J.L. (2012): "Las cooperativas ante la globalización: magnitudes, actividades y tendencias", Ekonomiaz, 79: 13-30.

MORALES, A.C. (2004): "La dirección en la empresa de trabajo asociado: una revisión de estudios empíricos", CIRIEC-España, Revista de Economía Pública, Social y Cooperativa, 48: 99-122.

OSTERBERG, P. \& NILSSON, J. (2009): "Members' perception of their participation in the governance of cooperatives: The key to trust and commitment in agricultural cooperatives", Agribusiness, 25: 181-197.

PÉROTIN, V. (2006): "Entry, exit and the business cycle: Are cooperatives different?", Journal of Comparative Economics, 34: 295-316.

PÉROTIN, V. (2015): "What Do We Really Know About Workers' Cooperatives?". En: ILO-ICA Research Conference on Cooperatives and the World of Work, 9-10 Noviembre 2015, Antalya (Turquía).

PETROU, A.P. (2009): "Foreign market entry strategies in retail banking: Choosing an entry mode in a landscape of constraints", Long Range Planning, 42: 614-632.

PODIVINSKY, J.M. \& STEWART, G. (2007): "Why is Labour-Managed Firm Entry So Rare? An analysis of entry using UK panel data", Journal of Economic Behavior \& Organization, 63: 177-192. 
QIAN, G. (2002): "Multinationality, product diversification and profitability of emerging US small and medium sized enterprises", Journal of Business Venturing, 17: 611-633.

ROTHSCHILD-WHITT, J. (1986): The Co-operative Workplace: Potentials and Dilemmas of Organisational Democracy and Participation, New York: Cambridge Press.

SANDERS, W.G. \& CARPENTER, M.A. (1998): "Internationalization and firm governance: The roles of CEO compensation, top team composition, and board structure", Academy of Management Journal, 41: 158-178.

SAPIENZA, H.J., AUTIO, E., GEORGE, G., et al. (2006): "A Capabilities Perspective on the Effects of Early Internationalization on Firm Survival and Growth", Academy of Management Review, 31(4): 914-33.

SHARMA, V.M. \& ERRAMILLI, M.K. (2004): "Resource-based explanation of entry mode choice", Journal of Marketing Theory and Practice, 12: 1-18.

SPEAR, R. (2004): "Governance in democratic member-based organizations", Annals of Public and Cooperative Economics, 75: 33-60.

TIHANYI, L., GRIFFITH, D.A. \& RUSSELL, C.J. (2005): "The Effect of Cultural Distance on Entry Mode Choice, International Diversification, and MNE Performance: A Meta-Analysis", Journal of International Business Studies, 36(3): 270-283.

VAN DER KROGT, D., NILSSON, J. \& HOST, V. (2007): "The impact of co-operatives' risk aversion and equity capital constraints on their inter-firm consolidation and collaboration strategies", Agribusiness, 23: 453-472.

VANEK, J. (1977): The Labor-Managed Economy, Ithaca, NY: Cornell University Press.

VARUM, C.A. \& ROCHA, V.C. (2011): "Do foreign and domestic firms behave any different during economic slowdowns?", International Business Review, 20(1): 48-59.

YIP, G.S., BISCARRI, J.G. \& MONTI, J.A. (2000): "The role of the internationalization process in the performance of newly internationalizing firms", Journal of International Marketing 8(3): 10-35. 
